\title{
Income and Wealth Distributions from Stochastic Strategy Minority Game
}

\author{
Asim Ghosh \\ Department of Computer Science \\ Aalto University School of Science \\ P.O. Box 15400, FI-00076 AALTO, Finland \\ asim.ghosh@aalto.fi \\ Soumyajyoti Biswas \\ Department of Dynamics of Complex Fluids \\ Max Planck Institute for Dynamics and Self-Organization \\ Am Fassberg 17, Göttingen-37077, Germany \\ soumyajyoti.biswas@ds.mpg.de
}

Received 14 November 2016

Accepted 8 December 2016

Published 19 January 2017

\begin{abstract}
We propose a version of the Minority Game where the agents at each time make investments either in terms of money or stock from their possessions. The amount of investments at each time step, and not the number of people opting for a choice, determines the 'minority'. The invested money is returned to the stock investors and the invested stock is returned to the money investors in proportion of their respective investments at each time. In this way, agents in the less investment side faces higher demand, and hence are in 'minority', receiving higher pay-off for their investments. This dynamics lead the 'market' to a self-organized state. We measure the distributions of income of the agents at every step and also the accumulated wealth, both of which have a stationary distribution. The distribution functions follow Pareto's law when the agents invest random fractions of their wealth. This reflects the role of heterogeneity in economic interactions.
\end{abstract}

Keywords: Minority game; stock market; income and wealth distribution; power law.

\section{Introduction}

The price fluctuations of a commodity in a market are the result of the imbalance in its demand and supply. Similarly, price fluctuation of a stock in a stock-market is the result of imbalance of traders' interests in buying and selling a stock. If more people

This is an Open Access article published by World Scientific Publishing Company. It is distributed under the terms of the Creative Commons Attribution 4.0 (CC-BY) License. Further distribution of this work is permitted, provided the original work is properly cited. 
are interested in selling than buying, then the price of that stock goes down and vice versa. It is therefore often compared with the Minority Game problem ${ }^{1-3}$ where the players are asked to make a choice between two options at each time step. The players ending up in the minority receive a positive pay-off, much like the smaller number of buyers in a market with more sellers.

The Minority Game is played between $N$ independent players making parallel decisions. The number $N$ is taken to be odd, to make an unambiguous minority at each step. The objective of the game is to divide the population almost equally between the two choices and hence to maximize the resource utilization and also to do that within a short time. This is a variant of the El Farol bar problem ${ }^{4}$ and has a vast literature in dealing with this objective that concerns with various strategies and their merits and demerits. In particular, stochastic strategies have been useful in maximizing the resource utilization ${ }^{5,6}$ (see Ref. 7 for a review).

Our first objective in this study is to define the minority in a more realistic way in terms of imbalance in demand and supply rather than just the number of people opting for a choice. In the traditional variant, it is effectively assumed that the selling and purchasing power of every agent is the same, which is far from realistic. It is the amount of stock to be sold and the amount of money involved in buying those stocks that determine the imbalance in demand and supply and hence the 'minority'.

Our second objective is to check the distributions of income and wealth (amount of stock and/or money) among the agents after the game is played repeatedly for a long time. The income and wealth distribution among people in a society is another long studied problem. Empirical laws, such as the Pareto's law, ${ }^{8}$ are known to be valid across many nations and at different times. ${ }^{9}$ It is therefore very natural to demand that a well known form of wealth exchange, such as the stock market dynamics, should respect such empirical laws. There have been very few attempts in the past along this line ${ }^{10,11}$ which at the end did not recover the wealth and income distributions very well.

In the following sections, we first define the new version of the Minority Game model and then discuss the nature of the income and wealth distributions in those models. Finally, we discuss some open questions and conclude.

\section{Model}

We consider the dynamics of a single stock and $N$ agents (see Fig. 1 for a schematic diagram) making repeated choice of either buying or selling that stock. The state of each agent (i) at every time step $(t)$ is given by three variables, the amount of stock $s_{i}(t)$ and the amount of money $m_{i}(t)$ she has at that time and a third variable that shapes the dynamics is the 'saving propensity' $\lambda_{i}$. This is similar to the case in the kinetic exchange models of wealth exchanges, ${ }^{12-15}$ where a saving propensity was introduced from a realistic perspective of that occurring in real life. At every time step, the agents must choose between buying (choice $\mathcal{B}$ ) or selling (choice $\mathcal{S}$ ). In other words, they invest a fraction of their money $\left[\Delta m_{i}=\left(1-\lambda_{i}\right) m_{i}(t)\right]$ or stock 


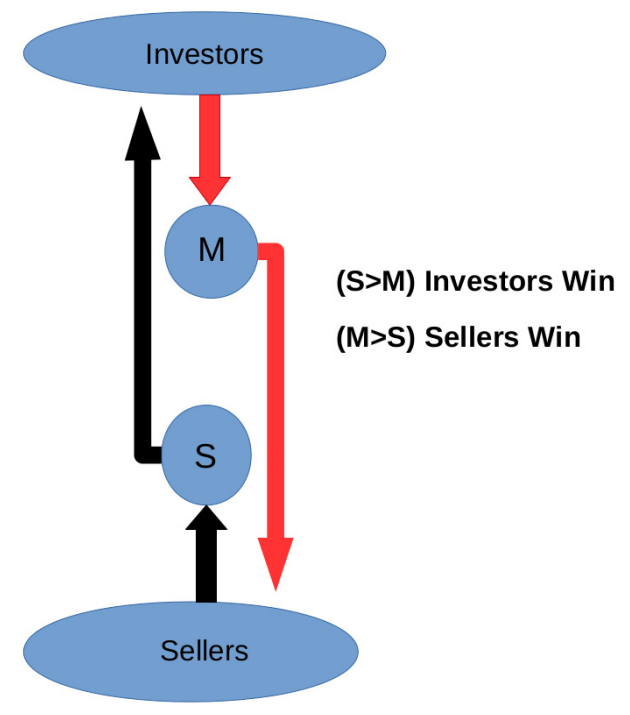

Fig. 1. Schematic diagram for the model. The group with smaller investment gets more return and hence the winner. Therefore, the group with smaller investment can be though of as the minority.

$\left[\Delta s_{i}=\left(1-\lambda_{i}\right) s_{i}(t)\right]$ at each step. Then, the total money invested $M(t)=$ $\sum_{i \in \mathcal{B}} \Delta m_{i}(t)$ is redistributed between the stock investors in the proportion of their investments, i.e., the agent with an investment of stock $\Delta s_{i}(t)$ will get back money $\delta m_{i}\left(\Delta s_{i}(t)\right)=M(t) \frac{\Delta s_{i}(t)}{S(t)}$, where $S(t)=\sum_{i \in \mathcal{S}} \Delta s_{i}(t)$. Similarly, for an agent investing money $\Delta m_{i}(t)$ there will be a return in terms of stock $\delta s_{i}\left(\Delta m_{i}(t)\right)=S(t) \frac{\Delta m_{i}(t)}{M(t)}$. Note that throughout this work we have considered the choice between buying and selling at each time to be completely random. Of course, this is not true in reality. However, optimization of this step is very different from the traditional Minority Game problem, since it is not the number of agents but their amount of investments that is determining the minority here.

The only parameter therefore, is the form of the saving propensity. In the following, we measure the distribution of stock and money among the agents at steady state for various form of saving propensity and also the distribution of 'income' at each time step of the agents (money earned by the agents at each step) who gets money. We consider the cases of constant saving fraction, where each agent saves a fraction $\lambda$ of her wealth and also the distributed savings, where the variables $\lambda_{i}$ are random for each agent but fixed in time. In all cases, however, we consider the saved value of saving propensity for a given agent for both stock and money.

Considering the fact that only a single stock is traded and also the choices are made randomly, this model is of course far from the reality. Nevertheless, the model in this simplest form gives many realistic features which we will discuss here. Further extensions are to be taken up elsewhere. 


\section{Results}

\subsection{Wealth distribution between the agents}

The agents only invest a fraction $\left(1-\lambda_{i}\right)$ of their stock or money each time and that is chosen randomly. Due to the presence of only one stock in the market, stock and money are symmetric in this case. Therefore, the wealth distribution can be measured by measuring the distribution of either one of those. After a sufficient relaxation time, the distribution function of stock/money comes to a steady state. Figure 2 depicts the results of such distributions for different constant values of $\lambda$ as well as when they are uniformly distributed in [0:1].

The case of zero saving leads to an absorbing state at the end. This is because, some agents can eventually get completely broken, and that fraction will subsequently increase, finally concentrating the entire wealth to one agent. For non-zero but fixed values of $\lambda$, the distribution is either exponential or gamma-like depending on whether $\lambda$ is less than or greater than 0.5 . This is somewhat surprising in the sense that even for a finite savings, most people have very low wealth, if $\lambda<0.5$ (see Fig. 3). This gives rise to a critical saving fraction $\lambda_{c}$ for the population to have zero density at zero wealth.

The most interesting case is the one with distributed saving propensity, i.e., the saving propensities $\lambda_{i}$ are different for each agent and are taken from a distribution uniform in [0:1]. It has two power-law regions, separated by the average wealth 1. This means, agents who are much richer than the average, follow a power law distribution with exponent -2 (this part can depend on the distribution of the saving propensities). This is in very good agreement with the empirical Pareto-law. Similarly as before, the average wealth and income increase with increasing saving propensities in the society (see Figs. 5 and 6).

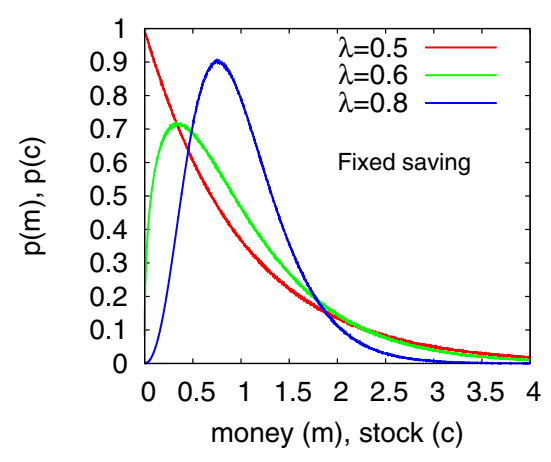

(a)

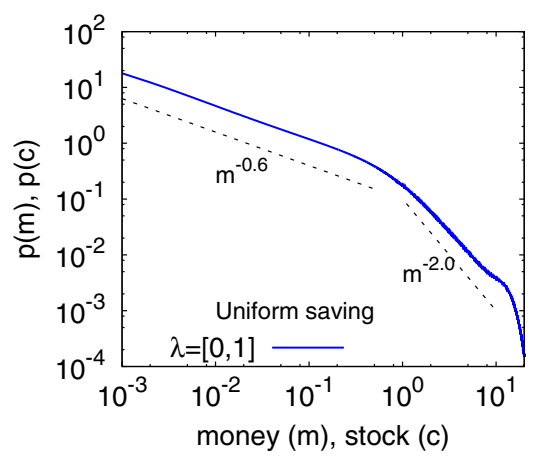

(b)

Fig. 2. The distribution of wealth (stock or money) among the agents, (a) when the saving propensity $\lambda$ has constant values and (b) when $\lambda_{i}$ are uniformly distributed in [0:1]. 


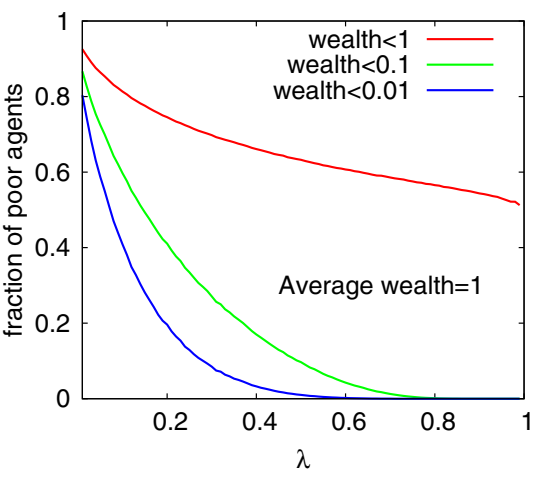

(a)

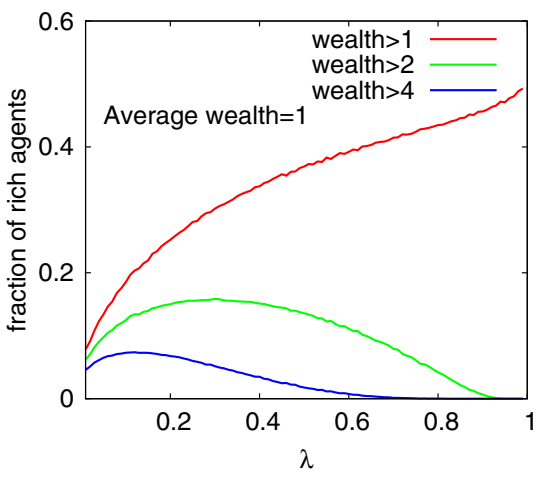

(b)

Fig. 3. For constant saving propensities, the fraction of agents having very low wealth (a) remains finite for almost upto $\lambda_{c} \approx 0.5$. The fraction of agents with very high income (b) also depends non-monotonically on $\lambda$. It can be seen that for low values of $\lambda$ the inequality is very high.

\subsection{Distribution of income}

The agents who invest (sell) stock, get back money in proportion of their investments. This constitute their 'income' at each time step. We measure the size distribution of these incomes in the steady state. Figure 4 represents such a distribution for fixed $\lambda$. It looks similar to the wealth distribution. In the same figure, we plot the Gini-index for different values of $\lambda$, which is a measure of the inequality in the income Ref. 16 (see also Ref. 17 for other measures and comparison with World Bank data).

For distributed saving, however, the income distribution (shown in Fig. 7) is different from the wealth distribution. Nevertheless, it has a power-law tendency before dropping off with a cut-off. The Gini-index for this case is about 0.54 and is in the range typically observed in different biased countries 18 .

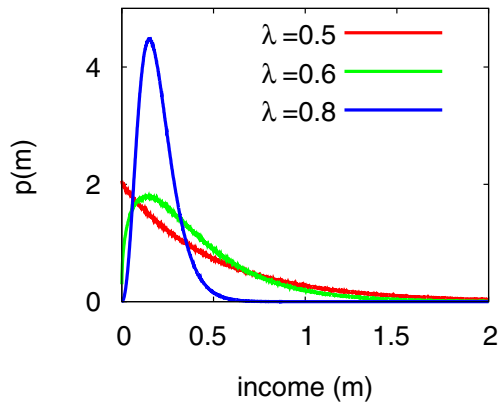

(a)

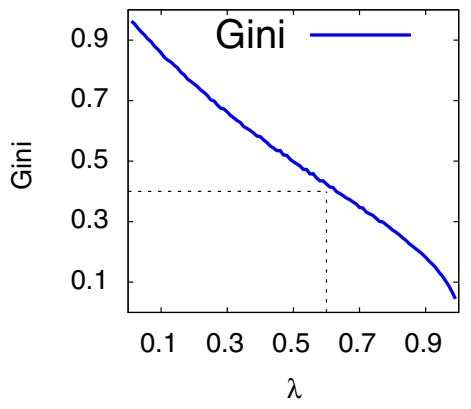

(b)

Fig. 4. (a) The distribution of income of the agents who are getting money in return, is shown when the saving propensities of the agents are constants. (b) The measurement of inequality in terms of Giniindex are shown for different values of saving propensity (see also Ref. 18 for the fairness bound of Gini index). 

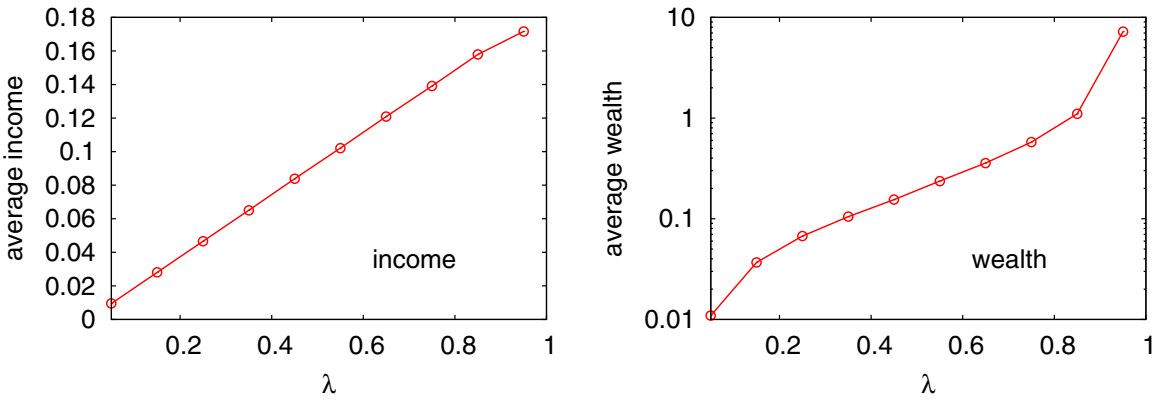

Fig. 5. For distributed saving propensities, the average income and wealth are plotted against different saving propensities in the society. It shows that for higher $\lambda$ the income and as well as the accumulated wealth increase.
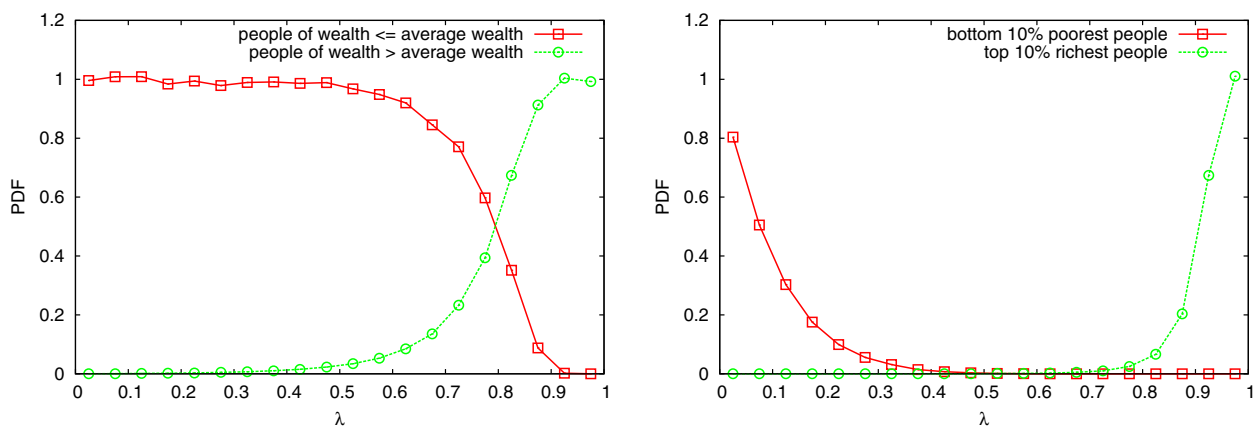

Fig. 6. For distributed saving propensities, the probability distributions for the saving propensities of the rich and poor sections of the society are plotted. On the left, the poverty line is just the average wealth, while on the right we define poor and rich as the bottom and top $10 \%$ of the society. In general, high savings lead to higher wealth.

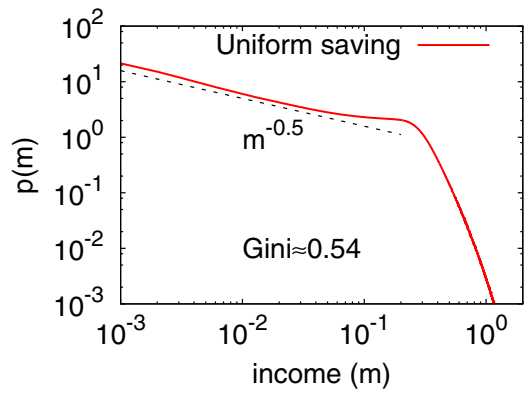

Fig. 7. The distribution of income when the saving propensity $\lambda_{i}$ are random variables among the agents taken from a uniform distribution in $[0: 1]$. 

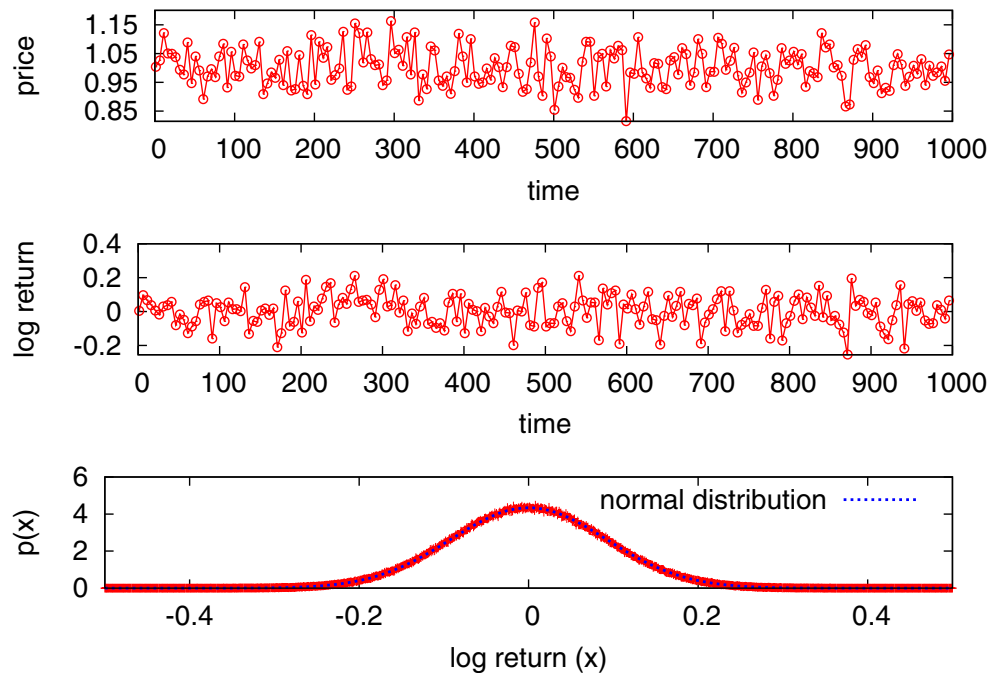

Fig. 8. The time series of prices, i.e., ratio of total stock and total money invested at a time is plotted for constant $\lambda=0.9$. The following panels show the log-return and the size distribution of the log-returns which matches with a normal distribution.

\subsection{Price fluctuation}

The price of the stock will fluctuate as the investments vary at each time. The price $S(t)$ is of course defined as the ratio of the total invested stock and total invested money at each time step. We compute the log-return $r=\log (S(t) / S(t-1))$ and the distribution of the log returns for both fixed (Fig. 8) and distributed saving (Fig. 9).
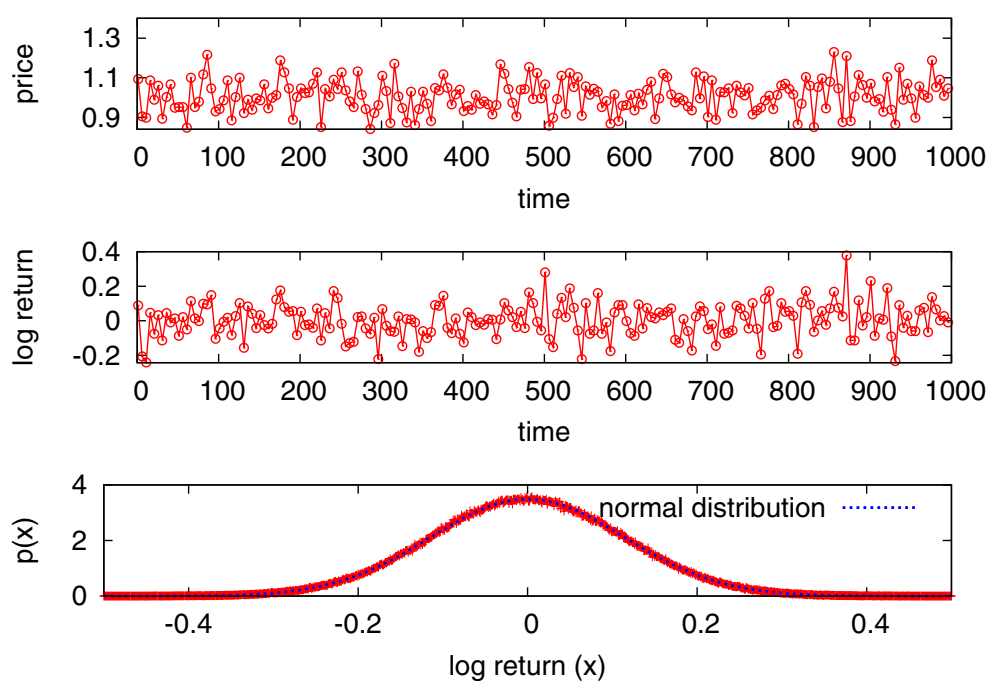

Fig. 9. The price fluctuations, log-return and its size distribution are plotted for uniformly distributed saving propensities. 
It matches with the normal distribution since the process here is completely random, i.e., there is no temporal correlation between the events. This is certainly not the case for the real stock markets, where the distribution differs from normal.

\section{Conclusions}

We have proposed a Minority-Game-like model for the stock market dynamics. The players have two choices of either investing money or stocks at each time step. Then, the invested money is returned to the stock investors and the invested stock is returned to the money investors in proportion to their respective investments. If the total invested stock is less than the total invested money, then the stock investors are in minority and receive a higher pay-off and vice versa. Therefore, the minority is determined by the amounts of investments and not by the number of investors. As a gross simplification, we have assumed all players to be random traders.

This simple model reproduces several features of income and wealth distributions. Particularly, when the agents invest a random fraction of their stock or money at each step (saving fractions drawn from uniform distribution), the steady state distribution of the accumulated wealth has a power-law. Furthermore, the distribution of income, i.e., the money earned by the stock investors at each step, is also a powerlaw. These features are in accordance with the Pareto's law, which is a widely held observation for various economies. The measure for income inequality, viz the Gini coefficient has a value $>0.4$ indicating a biased society. The bias is entering the model in the sense that the riches have most of the investment and therefore have most of the return. On the other hand, a poor agent does not win much even after ending up in the winning side. This effect accumulates in giving a biased wealth distribution (see Fig. 5). An obvious future direction is to investigate the effects of having rational agents and multiple types of stocks.

\section{References}

1. D. Challet and Y.-C. Zhang, Physica A 246 (1997) 407.

2. D. Challet and Y.-C. Zhang, Physica A 256 (1998) 514.

3. D. Challet, M. Marsili and Y.-C. Zhang, Minority Games (Oxford University Press, Oxford 2005).

4. W. Brian Arthur, Am. Econ. Rev. 84 (1994) 406.

5. D. Dhar, V. Sasidevan and B. K. Chakrabarti, Physica A 390 (2011) 3477.

6. S. Biswas, A. Ghosh, A. Chatterjee, T. Naskar and B. K. Chakrabarti, Phys. Rev. E 85 (2012) 031104.

7. A. Chakraborti, D. Challet, A. Chatterjee, M. Marsili, Y.-C. Zhang and B. K. Chakrabarti, Phys. Rep. 552 (2015) 1.

8. V. Pareto, Cours d'economie Politique (Rouge, Lausanne, 1897).

9. B. K. Chakrabarti, A. Chakraborti, S. R. Chakravarty and A. Chatterjee, Econophysics of Income and Wealth Distributions (Cambridge University Press, 2013).

10. K. H. Ho, F. K. Chow and H. F. Chau, Phys. Rev. E 70 (2004) 066110.

11. M. Tanaka-Yamawaki, Knowledge-Based Intelligent Information and Engineering Systems, Vol. 3681 (Springer-Verlag, 2005), p. 15. 
12. A. Chakraborti and B. K. Chakrabarti, Eur. Phys. J. B 17 (2000) 167.

13. A. Chatterjee, B. K. Chakrabarti and S. S. Manna, Physica A 335 (2004) 155.

14. A. Dragulescu and V. M. Yakovenko, Eur. Phys. J. B 17 (2000) 723.

15. V. M. Yakovenko and J. Barkley Rosser, Jr. Rev. Mod Phys. 81 (2009) 1703.

16. C. Gini, Econ. J. 31 (1921) 121.

17. A. Ghosh, A. Chatterje, J.-I. Inoue and B. K. Chakrabarti, Physica A 451 (2016) 465.

18. O. Flomenbom et al., The Gini of Fairness (this issue). 